\title{
Cryptotanshinone Induces Apoptosis and Autophagic Cell Death in Prostate Cancer Cells
}

\author{
Xiu-mei Liu', Yuan Fang ${ }^{1}$, Qian-qian Guo ${ }^{1}$, Ping Liu ${ }^{1}$ \\ ${ }^{1}$ Department of Clinical Pharmacology, People's hospital of Zhengzhou, \\ Zhengzhou, Henan, China.
}

Correspondence: Xiu-mei Liu, Department of Clinical Pharmacology, People's hospital of Zhengzhou, Zhengzhou, Henan, China. Email: liuxiumei1984@163.com

\begin{abstract}
Prostate cancer is one of the most common cancers and one of the leading causes of cancer-related death among men of all races. Complementary and alternative medicine is also used in the treatment of prostate cancer, thus, screening of natural products represents a promising strategy to develop potential anticancer drugs for prostate treatment. Cryptotanshinone, one of the major tanshinones isolated form Danshen, has been reported to inhibit cell proliferation and to induce cell death in various cancer cell lines, including those of colon, cervical, breast, etc. This study aims to explore the cytotoxicity of cryptotanshinone on human prostate cancer cell lines PC3 and DU145 as well as the underlying mechanisms of action. Our results demonstrate the selective cytotoxicity of cryptotanshinone in prostate cancer cells PC3 $\left(\mathrm{IC}_{50}=15.85 \mu \mathrm{M}\right)$ and DU145 $\left(\mathrm{IC}_{50}=7.59 \mu \mathrm{M}\right)$ as well as the underlying mechanisms of action, in particular the activation of both apoptosis and autophagic cell death. These findings suggested that cryptotanshinone is worthy to be further studied for its anticancer potential against prostate cancer.
\end{abstract}

Keywords: Cryptotanshinone; Apoptosis; Autophagy; Prostate cancer

http://mo.qingres.com

GOPEN ACCESS

DOI: $10.20900 / \mathrm{mo} .20170019$

Received: May 24, 2017

Accepted: July 28, 2017

Published: August 25, 2017

Copyright: @2017 Cain et al. This is an open access article distributed under the terms of the Creative Commons Attribution License, which permits unrestricted use, distribution, and reproduction in any medium, provided the original author and source are credited.

\section{INTRODUCTION}

Prostate cancer refers to the development of cancer in the prostate. It is one of the most common cancers and one of the leading causes of cancer-related death among men of all races ${ }^{[1]}$. In addition to surgery, chemotherapy and radiation, complementary and alternative medicine is also used in the treatment of prostate cancer, some examples include supplements like vitamins and herbs. Different naturalderived products and extracts including curcumin, glycyrrhetinic acid, capsaicin, genistein, berberine and green tea extract have been tested 
for their anticancer potential against prostate cancer ${ }^{[2]}$, suggesting that screening of natural products represents a promising strategy to develop potential anticancer drugs for prostate treatment.

Danshen is a Traditional Chinese Medicine isolated from the dried root of Salvia miltiorrhiza Bunge ${ }^{[3,4]}$. It is widely used in China for the prevention and treatment of cardiovascular diseases [5]. The anticancer potential of tanshinones, bioactive constitutes in Danshen, have also been reported previously, in particular in colon cancer cells and mouse xenograft models ${ }^{[6,7]}$. For example, the anticancer potential of dihydrotanshinone against colon cancer was reported in a HCT116 colon cancer cell xenograft model in mouse ${ }^{[8]}$. The studies by $\mathrm{Hu}$. et al also demonstrated the cytotoxicity of cryptotanshinone and dihydrotanshinone in various colon cancer cells, in particular in those cells with multidrug resistance ${ }^{[9,10]}$. A recent paper also reported the cytotoxicity of cryptotanshinone in multidrug-resistant colon cancer cells SW620 Ad300 and its induction of autophagic cell death in the cells ${ }^{[11]}$. However, the anticancer potential of cryptotanshinone on prostate cancer cells has not been well-studied yet. In this study, therefore, we aim to explore the cytotoxicity of cryptotanshinone on human prostate cancer cell lines PC3 and DU145 as well as the underlying mechanisms of action.

\section{MATERIALS AND METHODS}

\subsection{Materials}

Cryptotanshinone ( purity > $98 \%$ ) was obtained from Sigma-Aldrich Corporation (St. Louis, MO, USA). Eagle's minimum essential medium (EMEM), RPMI 1640 medium, fetal bovine serum (FBS) and penicillin-streptomycin were from Gibco (Carlsbad, CA, USA). All the primary antibodies were purchased from Cell Signaling Technology.<smiles>C[C@H]1COC2=C1C(=O)C(=O)c1c2ccc2c1CCCC2(C)C</smiles>

Fig. 1 Chemical structure of cryptotanshinone

\subsection{Cell culture}

Normal human prostate epithelial cells and human prostate cancer cell lines PC3 and DU145 were purchased from the American Type Culture Collection (Manassas, VA, USA). The cells were maintained in respective culture medium supplemented with $10 \% \mathrm{FBS}, 100 \mathrm{U} / \mathrm{mL}$ penicillin and $100 \mu \mathrm{g} / \mathrm{mL}$ streptomycin at $37^{\circ} \mathrm{C}$ in a humidified atmosphere with $5 \% \mathrm{CO}_{2}$.

\subsection{Cell viability assay}

Cell viability was measured by MTT assay. Briefly, cells were seeded into 96-well plates at a density of 5,000 cells/well and allowed to grow overnight. After treatment with cryptotanshinone for 24 hours, MTT $(0.5 \mathrm{mg} / \mathrm{mL})$ was added into each well and incubated at $37{ }^{\circ} \mathrm{C}$ for another 4 hours. Afterwards, $100 \mu \mathrm{L}$ DMSO was added and the absorbance was determined at $570 \mathrm{~nm}$.

\subsection{Apoptosis assay by flow cytometry}

Cells were seeded into 12 -well plates at a density of $1.0 \times 10^{5}$ cells $/ \mathrm{mL}$. After treatment with cryptotanshinone for 24 hours, cells were harvested and resuspended in buffer containing annexin $V$ and $\mathrm{PI}$. After incubation at room temperature for 15 minutes, cells were analyzed by a BDLSRFortessa Cell Analyzer (BD Biosciences, San Jose, CA, USA), measuring the fluorescence emission at $530 \mathrm{~nm}$ and $575 \mathrm{~nm}$ using $488 \mathrm{~nm}$ excitation. Data were analyzed using Flow Jo 7.6.1 software (Tree Star, Inc., Ashland, OR, USA).

\subsection{Western blot}

Cells were seeded into 6-well plates at a density of $1.0 \times 10^{5}$ cells $/ \mathrm{mL}$ and were harvested after treatment with cryptotanshinone for 24 hours. $30 \mu \mathrm{g}$ of protein were resolved by SDS-PAGE and transferred onto PVDF membranes (BioRad, Hercules, CA, USA). The membranes were incubated with different primary antibodies diluted in $5 \%$ BSA in washing buffer at $40 \mathrm{C}$ overnight. Afterwards, the membranes were incubated with HRP-conjugated secondary antibodies at room temperature for 2 hours. Chemiluminescent signals were developed with LumiGLO reagent and Peroxide (Cell Signaling Technology) and detected.

\subsection{Statistical analysis}


Statistical analysis of the data was done using Prism 5.0 (GraphPad Software, CA, USA). All the data were expressed as mean \pm SEM. The significance of difference between groups was estimated by t-test or one-way analysis of variance (ANOVA) followed by Dunnett's post-hoc test. $p$ value less than 0.05 was considered statistical significance.

\section{RESULTS}

\subsection{Cytotoxicity of cryptotanshinone on prostate cancer cells}

The cytotoxicity of cryptotanshinone on normal human prostate epithelial cells and human prostate cancer cell lines PC3 and DU145 was measured by MTT assay. After 24 hours treatment, cryptotanshinone showed almost no cytotoxicity on normal human prostate epithelial cells (Fig. 2A). However, the viability of human prostate cancer cell lines PC 3 and DU145 was significantly decreased by cryptotanshinone (Fig. 2B and 2C). The IC50 values of cryptotanshinone on PC3 and DU145 cells were $15.85 \mu \mathrm{M}$ and $7.59 \mu \mathrm{M}$, respectively. These data suggest the selective toxicity of cryptotanshinone on human prostate cancer cells. Since DU145 cells were more sensitive to cryptotanshinone in terms of the cytotoxicity, this cell line was used for the following study to elucidate the mechanisms of action of cryptotanshinone.
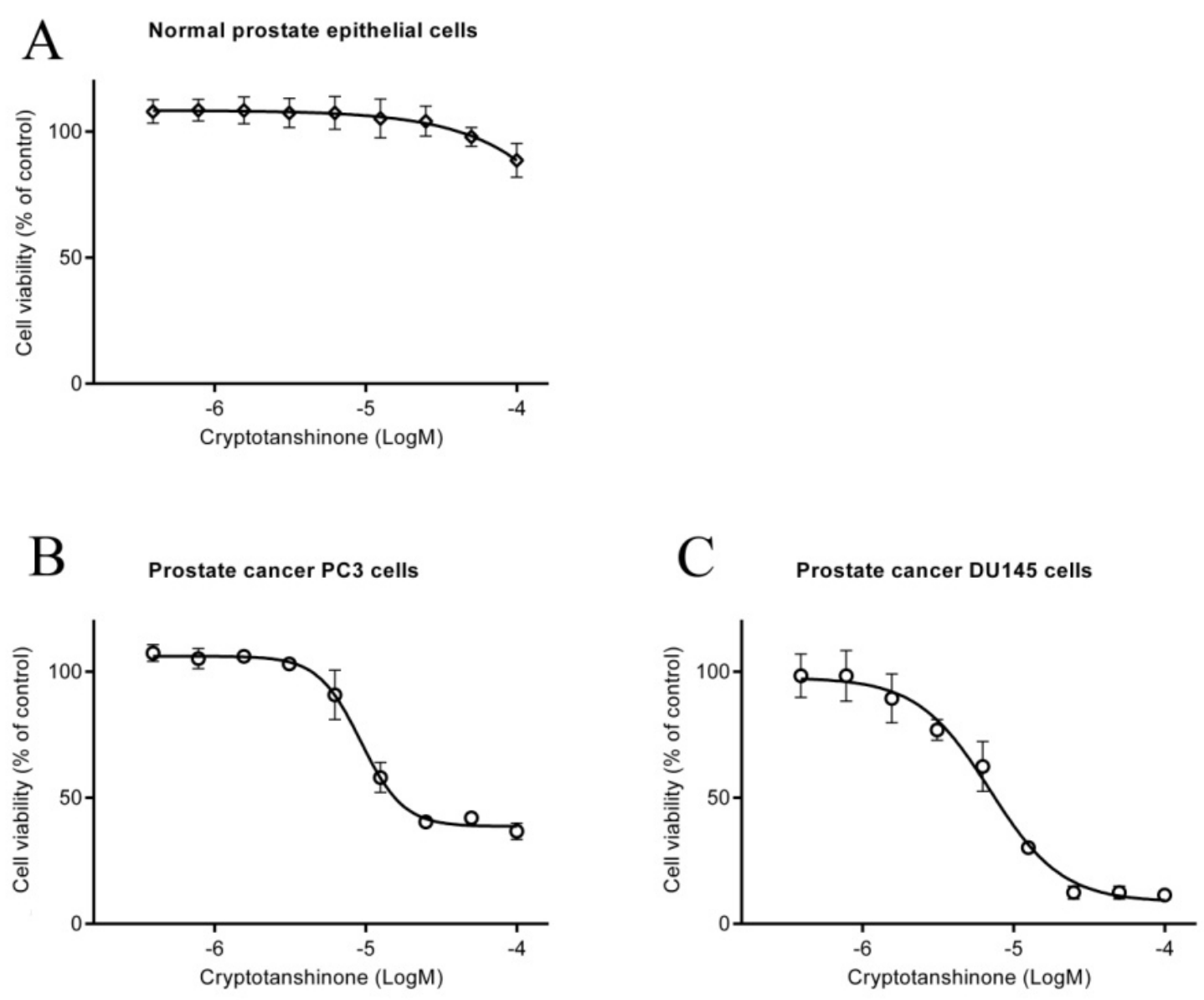

Fig. 2 Cell viability of normal prostate epithelial cells. (A) prostate cancer cells PC3 (B) and DU145 (C) after treatment with cryptotanshinone for 24 hours. 


\subsection{Cryptotanshinone induced apoptosis in DU145 cells}

To further explore the mechanisms through which cryptotanshinone caused cytotoxicity in prostate cancer cells, the level of apoptosis, type I programmed cell death, was examined in DU145 cells after treatment with cryptotanshinone for 24 hours. As shown in Fig. $3 \mathrm{~A}$ and $3 \mathrm{~B}$, annexin $\mathrm{V}$ and PI double staining indicated that cryptotanshinone induced significant apoptosis in DU145 cells, including $\sim 19.4 \%$ early stage apoptosis and $\sim 35.2$ $\%$ late stage apoptosis. Western blot analysis also showed that after cryptotanshinone treatment the protein levels of pro-apoptotic protein Bax and anti-apoptotic protein $\mathrm{Bcl}-2$ were increased and decreased, respectively. Moreover, significant activation of caspase- 3 was also detected in DU145 cells after treatment with cryptotanshinone for 24 hours (Fig. 3C). Taken together, the data demonstrate that cryptotanshinone induces significant apoptosis in DU145 cells, which contributing to its cytotoxic action on prostate cancer cells. Moreover, this finding provides a basis for nextstep expanding studies on whether cryptotanshinone can induce p53 expression because that Bax is substrate of it and cleaves caspase 8 and 9 because cryptotanshinone-induced apoptosis could be resulted from extrinsic or intrinsic pathway.
A

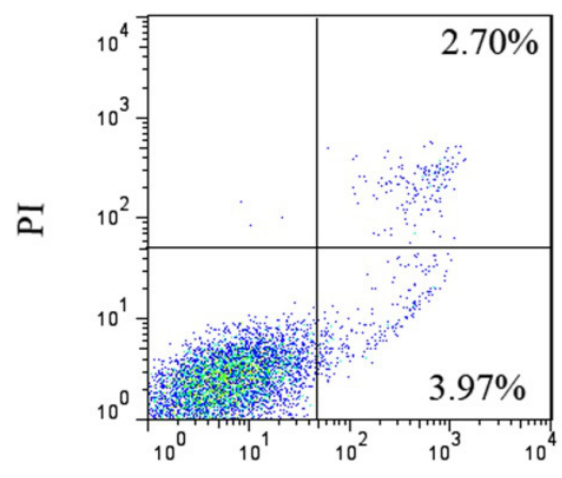

Cryptotanshinone $(10 \mu \mathrm{M})$

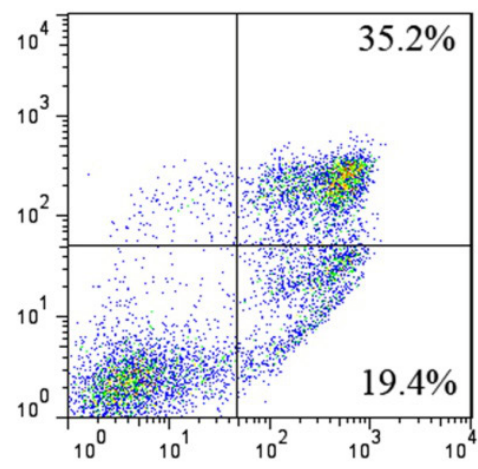

Annexin V

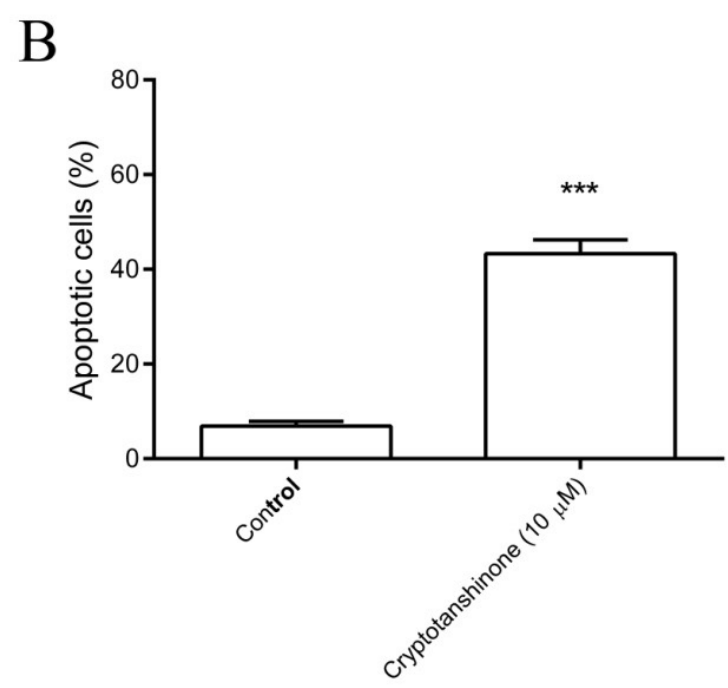

C Cryptotanshinone $\quad-\quad+$

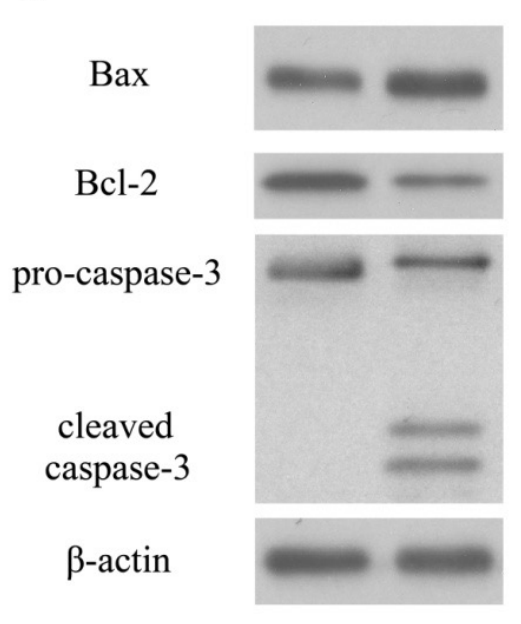

Fig. 3 Cryptotanshinone induced apoptosis in DU145 cells. (A) Apoptosis induced by cryptotanshinone in DU145 cells by annexin V and PI double staining. (B) Statistical analysis of the level of apoptosis in DU145 cells. ${ }^{* * *} p<0.001$, compared with DMSO group. (C) Western blot analysis showing the protein expression of apoptosis-related proteins in DU145 cells after cryptotanshinone treatment. 


\subsection{Cryptotanshinone induced autophagic cell death in DU145 cells}

In addition to apoptosis, autophagy, type II programmed cell death, may also play a role in leading to cell death. In this regard, the level of autophagy was examined in DU145 cells after treatment with cryptotanshinone, with an aim to see if autophagy is involved in the cytotoxicity of cryptotanshinone on prostate cancer cells. As shown in Fig. 4A, the protein level of microtubule-associated protein light chain3 (LC3)B-II, an autophagosome membrane protein and autophagy marker, was highly up-regulated by cryptotanshinone in DU145 cells after 24 hours exposure. These data demonstrated the activation of autophagy by cryptotanshinone in
DU145 cells. Since autophagy may either protect the cells from stress or promote cell death, it is necessary to examine the role of cryptotanshinoneinduced autophagy in DU145 cells. In the presence of chloroquine (CQ), an autophagy inhibitor, the cytotoxicity of cryptotanshinone on DU145 cells was weaker when compared with cryptotanshinone alone group (Fig. 4B), suggesting that autophagy induced by cryptotanshinone in DU145 cells also led to cell death, therefore contributing to the cytotoxicity of cryptotanshinone on prostate cancer cells. Moreover, those findings provide clues to further test the alterations of more autophagy markers such as Atg7, BIF1 or Gtr2 in cryptotanshinone-treated prostate cancer cells.

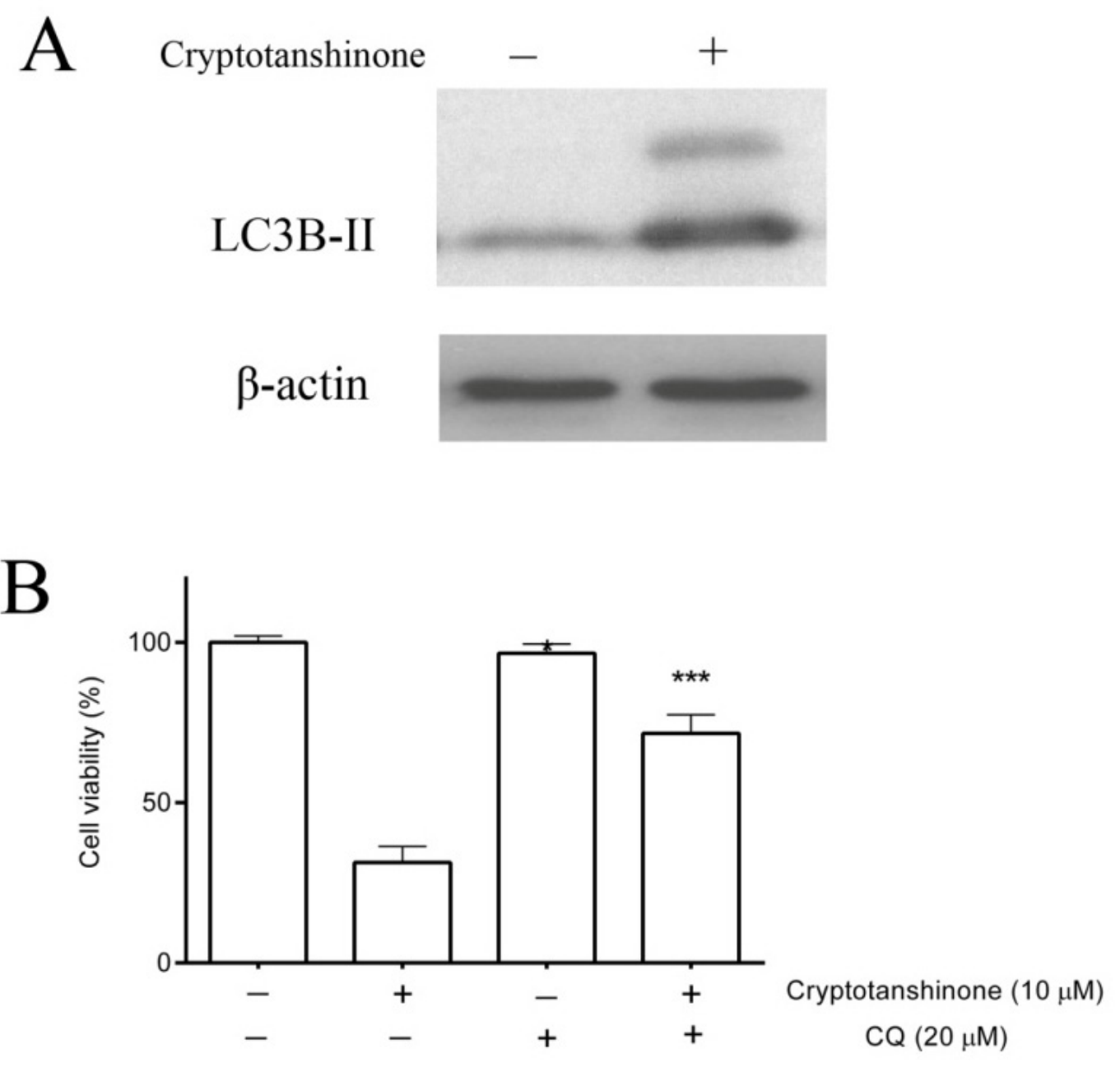

Fig. 4 Cryptotanshinone induced autophagic cell death in DU145 cells. (A) Protein expression of LC3BII in DU145 cells after treatment with cryptotanshinone for 24 hours. (B) Cytotoxicity of cryptotanshinone in the presence of $\mathrm{CQ}$, an autophagy inhibitor. ${ }^{* * *} p<0.001$, compared with cryptotanshinone alone group. 


\section{DISCUSSION}

Cryptotanshinone, one of the major tanshinones isolated form Danshen, is a cell-permeable diterpene quinone. It has been reported to inhibit cell proliferation and to induce cell death in various cancer cell lines, including those of colon, cervical, breast, etc. Some underlying mechanisms of action of cryptotanshinone include the induction of cell cycle arrest and activation of apoptosis. For instance, cryptotanshinone induced a G2/M phase cell cycle arrest in melanoma (B16) cells via its upregulation of cyclin A1, cyclin B1 and Cdc25c ${ }^{[12]}$. The compound is also able to induce apoptosis by activating mitogen-activated protein kinase (MAPK) cascade in hepatoma (HepG2) and breast carcinoma (MCF7) cells ${ }^{[13]}$. Besides, cryptotanshinone has also been reported to sensitize drug resistant prostate cancer DU145 cells to Fas (APO1/CD95)-mediated apoptosis as well as several chemotherapeutic agents including cisplatin, TNF- $\alpha, 5-F U$ and doxorubicin ${ }^{[14]}$. These previous findings suggested the anticancer potential of cryptotanshinone against different cancers, via its versatile mechanisms of action. Based on the results in this study, cryptotanshinone was found to induce both apoptosis and autophagic cell death in human prostate cancer cell lines PC3 and DU145. More importantly, this compound showed almost no cytotoxicity on normal human prostate epithelial cells. The selective cytotoxicity of cryptotanshinone towards prostate cancer cells made it a potential candidate for the further development of anticancer drugs.

Apoptosis and autophagy are two types of programmed cell death. The functional relationship between apoptotic and autophagic signaling pathways is complicated in cancer cells. The upstream triggers including death-associated protein kinase (DMPK), reactive oxygen species (ROS), ceramide, p53 and $\mathrm{Ca}^{2+}$ are able to induce either apoptosis or autophagy in the cells. Some feedback loops and crosstalk also exist between apoptotic and autophagic signaling pathways, which are frequently altered in cancer cells ${ }^{[15]}$. Our results demonstrate the activation of both apoptosis and autophagic cell death by cryptotanshinone in DU145 cells. However, the alterations of apoptotic and autophagic signaling pathways by cryptotanshinone were not fully studied. Whether cryptotanshinone could activate common upstream triggers of both signaling pathways still needs to be elucidated in the future.

Regarding the in vivo biological activity of cryptotanshinone, one major concern is the low bioavailability of the compound. Different formulations of cryptotanshinone were made with an aim to improve its plasma concentration in the body. For example, a different group developed a hydroxylpropyl- $\beta$-cyclodextrin-included complex of cryptotanshinone. Using this complex, the oral bioavailability of cryptotanshinone was increased by 2.5 -fold in rats when compared with the parent cryptotanshinone ${ }^{[16]}$. Therefore, such kind formulations of cryptotanshinone should be considered in the in vivo studies of cryptotanshinone in the future.

In conclusion, this study demonstrates the selective cytotoxicity of cryptotanshinone in prostate cancer cells as well as the underlying mechanisms of action, in particular the activation of both apoptosis and autophagic cell death. These findings suggested that cryptotanshinone is worthy to be further studied for its anticancer potential against prostate cancer.

\section{REFERENCES}

1. Cooperberg MR. Prostate cancer: a new look at prostate cancer treatment complications. Nat Rev Clin Oncol. 2014; 11(6): 304-305.

2. Kallifatidis G, Hoy JJ, Lokeshwar BL. Bioactive natural products for chemoprevention and treatment of castration-resistant prostate cancer. Semin Cancer Biol. 2016; 40-41: 160-169.

3. $\mathrm{Hu} \mathrm{T}$, Cho $\mathrm{CH}$. Potential Applications of Tanshinones in Gastrointestinal and Hepatic Diseases. J Biomol Res Ther. 2013; 2: 110.

4. Zhou $X$, Wang $Y, H u ~ T$, Or PM, Wong J, Kwan YW, Wan DC, Hoi PM, Lai PB, Yeung JH. Enzyme kinetic and molecular docking studies for the inhibitions of miltirone on major human cytochrome P450 isozymes. Phytomedicine. 2013; 20(3-4): 367-374.

5. Hu T, Zhou X, Wang L, Or PM, Yeung JH, Kwan $\mathrm{YW}$, Cho $\mathrm{CH}$. Effects of tanshinones from Salvia miltiorrhiza on CYP2C19 activity in human liver microsomes: enzyme kinetic and molecular docking studies. Chem Biol Interact. 2015; 230: 1-8.

6. Wang L, Yeung JH, Hu T, Lee WY, Lu L, Zhang L, Shen J, Chan RL, Wu WK, Cho CH. Dihydrotanshinone induces p53-independent but ROS-dependent apoptosis in colon cancer cells. Life Sci. 2013; 93(8): 344-351.

7. Wang L, Hu T, Shen J, Zhang L, Li LF, Chan $\mathrm{RL}$, Li MX, Wu WK, Cho $\mathrm{CH}$. Miltirone induced mitochondrial dysfunction and ROS-dependent apoptosis in colon cancer cells. Life Sci. 2016; 151: 224-234. 
8. Wang L, Hu T, Shen J, Zhang L, Chan RL, Lu $\mathrm{L}$, Li M, Cho CH, Wu WK. Dihydrotanshinone I induced apoptosis and autophagy through caspase dependent pathway in colon cancer. Phytomedicine. 2015; 22(12): 1079-1087.

9. Hu T, To KK, Wang L, Zhang L, Lu L, Shen $\mathrm{J}$, Chan RL, Li M, Yeung JH, Cho CH. Reversal of P-glycoprotein (P-gp) mediated multidrug resistance in colon cancer cells by cryptotanshinone and dihydrotanshinone of Salvia miltiorrhiza. Phytomedicine. 2014: 21(11): 1264-1272.

10. Hu T, Wang L, Zhang L, Lu L, Shen J, Chan $\mathrm{RL}$, Li M, Wu WK, To KK, Cho CH. Sensitivity of apoptosis-resistant colon cancer cells to tanshinones is mediated by autophagic cell death and p53-independent cytotoxicity. Phytomedicine. 2015: 22(5): 536-544.

11. Xu Z, Jiang $H$, Zhu $Y$, Wang $H$, Jiang J, Chen L, $\mathrm{Xu} \mathrm{W}, \mathrm{Hu} \mathrm{T}$, Cho $\mathrm{CH}$. Cryptotanshinone induces ROS-dependent autophagy in multidrugresistant colon cancer cells. Chem Biol Interact. 2017; 273: 48-55.

12. Chen L, Zheng SZ, Sun ZG, Wang AY, Huang $\mathrm{CH}$, Punchard NA, Huang SL, Gao X, Lu $Y$. Cryptotanshinone has diverse effects on cell cycle events in melanoma cell lines with different metastatic capacity. Cancer Chemother Pharmacol. 2011; 68(1): 17-27.

13. Park IJ, Kim MJ, Park OJ, Choe W, Kang I, Kim $\mathrm{SS}$, Ha J. Cryptotanshinone induces ER stressmediated apoptosis in HepG2 and MCF7 cells. Apoptosis. 2013; 17(3): 248-257.

14. Park IJ, Kim MJ, Park OJ, Park MG, Choe W, Kang I, Kim SS, Ha J. Cryptotanshinone sensitizes DU145 prostate cancer cells to Fas(AP01/CD95)-mediated apoptosis through $\mathrm{Bcl}-2$ and MAPK regulation. Cancer Lett. 2010; 298(1): 88-98.

15. Hu T, Li Z, Gao CY, Cho CH. Mechanisms of drug resistance in colon cancer and its therapeutic strategies. World J Gastroenterol. 2016; 22(30): 6876-6889.

16. Pan $Y$, Bi HC, Zhong GP, Chen X, Zuo Z, Zhao LZ, Gu LQ, Liu PQ, Huang ZY, Zhou SF, Huang M. Pharmacokinetic characterization of hydroxylpropyl-beta-cyclodextrin-included complex of cryptotanshinone, an investigational cardiovascular drug purified from Danshen (Salvia miltiorrhiza). Xenobiotica. 2008; 38(4): 382-398. 